\title{
EXPLORING THE ROLE OP PUNCTUATION IN PARSING NATURAL TEXT
}

\author{
Bernard E M Jones
}

\author{
Centre for Cognitive Science, University of Edinburgh, Edinburgh Fir8 9IW, Scotland
}

Jimail: bernie@cogsci.ed.ac.uk

\begin{abstract}
Few, if any, current NLP systems make any significant use of punctuation. Intuitively, a treatment of punctuation seems necessary to the analysis and production of text. Whilst this has been suggested in the fields of discourse structure, it is still unclear whether punctuation can help) in the syntactic ficld. This investigation attempts to answer this question by parsing some corpus-based material with two similar grammars -.. one including rules for punctuation, the other ignoring it. The punctuated grammar significantly ont-performs the unpunctrated one, and so the conclusion is that punctuation can play a useful role in syntactic processing.
\end{abstract}

\section{INTRODUCTION}

There are no current text based natural language analysis or generation systems that make full use of punctuation, and while there are some that make limited use, like the J'ditor's Assistant [Dale 1990], they tend to be the exception rather than the rule. Instead, punctuation is usually stripped ont of the text before processing, and is not inclucled in gencrated text.

Intuitively, this seems very wrong. Punctuation is such an integral part of written language that it is difficult to imagine naturally producing any significant body of unpunctuated text, or being; able to easily understand any such body of text.

Towever, this is what has been done in the computational linguistics field. 'T'he reason that it hats al ways been too difficult to incorporate any coherent account of punctuation into any system is because no such coherent account exists.

Punctuation has long been consiclered to be intimately related to intonation: that is that different punctuation marks simply give the reacler cues as to the possible prosodic and pausal characteristics of the text [Markwardt, 1942]. This claim is questioned by Nunberg [1.990], since such a transcriptional view of punctuation is theoretically uninteresting, and also correlates rather badly with intonation in any case.

However, even if we recognise that punctuation fulfils a linguistic role of its own, it is by no means clear how this role is defined. Since there is still no concise linguistic account of the function of punctu- ation, wo have to rely mainly on personal intuitions. 'This in tum introduces new problems, since there is a great cleal of idiosyncrasy associated with the use of punctuation marks. Whilst most people may agree on core situations in which use of a given punctuation mark is desirable, or even necessary, there are still many siluations where their use is less clear.

In his recent review, IIumphreys [1993] suggests that accounts of punctuation fall into three categories: "I'he first ... is selflessly dedicated to the task of bringing Punctuation to the Peasantry ...'The second sort is the Style Guide, written by editors and printers for the private pleasure of fellow professionals ... The third, on the linguistics of the punctuation system, is much the rarest of all."

'Thus whilst we do not really want to rely on publishers' style guides, since the accounts of punctuation they contain are rather too proscriptive and concentrate on the use of punctuation rather than its meaning, the academic accounts of punctuation are far from numerous. In the work of Dale [1991], the potential of punctuation in the field of discourse and natural language generation is explored. However, little mention is made anywhere of the role of punctuation within a syntactic framework. Therefore the current investigation tries to determine whether taking consideration of punctuation can further the goals of syntactic analysis of natural language.

\section{PUNC'TUATION}

Junchualion, ats we consider it, can be defined as the central part of the range of non-lexical orthography. Although arguments could be mate for including the sub-lexical marks (e.g. lypplsens, apostrophes) and structural marles (e.g. bullets in ilemisations), they are excluded since they tend to be lexicalised or rather difficult to represent, respectively. Indecd, it is difficult to imagine the representation of strue. tural punctuation, other than through the use of some special structural description languagesuch as SGMI.

Within our definition of punctuation then, we find broadly three types of mark: delimiting, separating and disambigualing, as deseribed by Nunberg [1990]. Some marks, the comma especially, fall into multiple categories since they can have different roles, and the categories each perform distinct linguistic functions.

belimiters (e.g. comma, dash, parenthesis) occur to either side of a particular lexical expression to remove that expression from the immediate syntactic 
context of the surrounding sentence (1). The delimited phrase acts as a modificr to the adjacent phrase instead.

(1) John, my friend, fell over and died.

Separating marks come between similar grammatical items and indicate that the items form a list (2). They are therefore similar to conjunctions in their behaviour, and can sometimes replace conjunctions in a list.

\section{(2) I came, I saw, I conquered.} I want butter, eggs and flour.

Disambiguating marks, usually commas, occur where an unintentional ambiguity could result if the marks were not there (3), and so perhaps illustrate best why the use of punctuation within NI, systems could be beneficial.

(3) Earlier, work was halted.

In addition to the nature of different punctuation marks, there are several phenomena described by Nunberg [1990] which it is useful to consider beforo implementing any treatment of punctuation:

Point absorption: strong point symbols (comma, dash, semicolon, etc.) absorls weaker adjacent ones (4). Commas are least powerful, and periods ${ }^{1}$ most powerlul;

(4) It was John, my friend.

Bracket absorption: commas and dashes are removed if they occur directly before an end quote or parenthesis (5);

(5) $\ldots$ (my brother, the teacher)...

Quote transposition: punctuation directly to the right of an end quote is moved to the left of that character (6). This phenomenon occurs chicfly in American English, but can occur generally;

(6) He said, "I love you."

Graphic absorption: orthographically, but not linguistically, similar coincident symbols are absorbed (7). Thus the dot marking an abbreviation will absorb an adjacent period whereas it would not absorb an adjacent comma.

(7) I work for the C.I.A., not the F.B.I.

In addition to the phenomena associated with the interaction of punctuation, there are also distinct phenomena observable in the interaction of punctuation and lexical expressions. Thus delimited plırases cannot immediately contain delimited phrases of the

\footnotetext{
${ }^{1}$ Throughout this paper I shall refer to sentence-final dots as periods rather than full-stops, to avoid confusion.
}

same type (the sole exception may be with parentheticals, though many people object to nested parentheses) and adjuncts such as the colon-expansion cannot contain further similar adjuncts. Therefore, in the context of colon and semicolon scopjing, $(8)$ is ambiguous, but (9) is not.

(8) words: words; words

(9) words : words; words : words .

\section{THE GRAMMAR}

Recognition of punctuational phenomena does not imply that they can be successfully encoded into a NI. grammar, or whether the use of such a punctuated grammar will result in any analytical advantages.

Nunberg [1990] advocates two separate grammars, operating at different levels. A lexical grammar is proposed for the lexical expressions occurring between punctuation marks, and a text grammar is proposed for the structure of the punctuation, and the relation of those marks to the lexical expressions they separate. The text grammar has within it distinct levels, such as phrasal and clansal, at which distinct punctuational phenomena can occur.

This should, in theory, make for a very neat system: the lexical syntiactic processes being kept separate from those that handle punctuation. llowever, in practice, this system seems unlikely to succed since in order to work, the lexical expressions that occur between punctuation marks must carry additional information about the syntactic categories occurring at their edges so that the text grammar can constrain the function of the punctuation marks.

For example, if a sentence inchudes an itemised noun phrase (10), the lexical expression before the comma must be marked as ending wilh a noun phrase, and the lexical expression after the comma must be makked as starting with a noun phase. A rule in the text grammar could then process the separating comma as it clearly comes between two similar syntactic elements.

\section{(10) We likes Willy, Jan and Tom. [end: np] [start: ne]}

Ilowever, as (11) shows, the separating comma concept could require information about the categories at arbitrarily deep levels occurring at the onds of lexical expressions surrounding punctuation marks.

(11) I like to walk, skip, and rurt.

I like to walk, to skip, and to rum.

I like to walk, like to skip, but late to run.

Even with the above edge-category information, the parsing process is not necessarily made any easier (since often the full partial parses of all the separate expressions have to be held and joineci). Therefore we scem to be at no advantage if we use this approach. 
In addition, it is difficult to imagine what linguistic or psychological motivation such a separation of punctuation from lexical text could hold, since it seems rather unlikely that people process punctuation at a separate level to the text it surrounds.

Hence it secms more sensible to use an integrated grammar, which handles both words and punctuation. This lets us describe the interaction of punctuation and lexical expressions far more logically and concisely than if the two were separated. Good examples of this are disambiguating commas; in it unified grammar we can simply write rules with an optional comma among the danghters (12).

(12) $\mathrm{s} \rightarrow \mathrm{np}$ (comma) vp. $\mathrm{s} \rightarrow \mathrm{pP}$ (comma) s.

A feature-based tag grammar was written for this investigation (based loosely on onc written by Briscoe and Waegner [1.992]), and used in conjumction with the parser inclucled in the Alvey 'I'ools' Grammar Development Fnvironment (GDIi) [Carroll et al, 1991], which allows for rapid prototyping and easy analysis of parses. It should be stressed that this grammar is solely one of tags, and so is not very detailed syntactically.

In order to handle the additional complications of punctuation, the notion of stoppedness of a category has been introduced. Tlus every category in the grammar has a stop feature which describes the punctuational character following it (13), and defiults to [st -] (unstopped) if there is no such character.

(13) the man, $=$ [st $\mathrm{c}]$

$$
\text { with the flowers. }=[\text { st } 1]
$$

Since the rules of the grammar further clictate that the mother category inherits the stop value of its rightmost daughter, only rules to specifically add punctuation for categories which could be lexicalised are necessary. 'Thus a rule for the additional of a punctuation mark after a lexicalised nom would be as in (14). (The calligraphic letters represent unification variables.)

$$
\text { (14) } \mathrm{n} 0[\text { st } S] \rightarrow \mathrm{n} 0[\text { st }-][\text { punc } S]
$$

We can then specify that top level categories must be [st f] (period), that items in a list should be [st c] (comma), etc. In rules where we want to force a particular punctuation mark to the right of a category, that mark can be included in the rule, with the preceding category unstopped: (15) illustrates the addition of a comma-delimited noun phrase to a noun phrase. Specifically mentioning the punctuation mark prevents the delimited phrase from being unstopped, resulting in an unstopped mother category. Notc that the phenomenon of point absorption has been captured by unifying the value of the st feature of the mother and the identity of the final punctuation mark. I'hus the possible values of st are all the possible values of punc in addition to [st -$]$.
(15) $n p[s t S] \rightarrow n p[s t$ c] $n p[$ st $\cdots][$ punc $S]$.

'Thus the stop feature seems sufficient to cope with the punctuational phenomena introduced alsove. In order to incorporate the plenomena of interaction between punctuation and lexical expressions (e.g. preventing immediate nesting of similar delimited phrases), we need to introduce at small number of additional features into the grammar. If, for example, we make a comma-delimited noun phrase [cm +$]$, we can then stipulate that auy nom pluase that includes a comma-delimited phrase has the feature [cm --], so that the two camnot unify (16). Note that the unification of mother and right-most daughter stop values is omitted for clarity of presentation.

\section{(16) $n p\left[\mathrm{~cm}^{-}\right]-+\mathrm{np}[\mathrm{st} \mathrm{c}] \mathrm{np}[\mathrm{cm}++$, st -$]$}

We can incorporate the relative scoping of colons and semicolons, as discussed previously, into the grammar very easily too. The semicolon rule (17) accepts any value of co in its arguments, but the colon rule (18) only accepts [co-]. The mother category of the colon rule bears the feature [co t-] to prevent inclusion into further colon-baring sentences. Note that there are more versions of the colon rule, which deal with diflerent constituents to either side of the colon, and also that, since the GDE does not permit the disjunction of feature values, the semicolon rule is merely an abbreviation of the multiple rules recuired in the grammar. Stop unification is again omitted.

(17) $s[\cos (\mathcal{A} \vee B)] \rightarrow \mathrm{s}[\operatorname{co} \mathcal{A}$, st sc $] \mathrm{s}[\mathrm{co} B]$.

(18) $s[\mathrm{co}+] \rightarrow s[\mathrm{co}-$, st $\mathrm{co}] \mathrm{s}[\mathrm{co}-]$.

Ilence the inchusion of a lew simple extra fatures in in normal grammar has achieved an acceptable treatment of punctuational phenomena. Since this work only represents the initial steps of provicling a full and proper account of the role of punctuation, no clitims are made for the theoretical validity or completeness of this approach!

\section{TIE CORPUS}

For the currem investigntion it. was necessary to use a corpus sulficicutly rich in punctuation to illustrate the possible advantages or disadvantages of utilising punctuation within the parsing process. Obviously a sentence which includes no punctuation will be equally difficult to parse with both punctuated and unjunchated grammars. Similarly, for sentencess including only one or two marks of punctuation, the use of punctuation is likely to be rather procedural, and hence not necessarily very revealing.

Therefore the tagged Spoken linglish Corpus was chosen ['Laylor \& Knowles, 1988]. 'I'his features some very long sentences, and includes rich and varied punctuation. Since the corpus has been punetuated manually, by several different people, some idiosyncrasy occurs in the punctuational style, but there is 
little punctuation which would be deemed inappropriate to the position it occurs in.

A subset of 50 sentences was chosen from the whole corpus. Between them these sentences include material taken from news broadcasts, poetry readings, weather forecasts and programme reviews, so a wide variety of language is covered.

The lengths of the sentences varied from 3 words to 63 words, the average being 31 words; and the punctuational complexity of the sentences varied from one mark (just a period) to 16 marks, the average being 4 punctuation marks. A sample tagged sentence is shown in (19), where $f s$ denotes a period.

\section{(19) Their_APP\$ meeting_NN1 involves_VVZ a_ATI} kind_NN1 of_lO life_NN1 swar,_NN1 fs_ES

The punctuated grammar, developed with this subset of the corpus, was used to parse the corpus subset, and then an unpunctuated version of the same grammar was used to parse the same subset. The reason that testing was performed on the training corpus was that, in the alssence of a complete treatment of punctuation, the punctuational phenomena in the training corpus were the only ones the grammar could work with, and although they included almost all of the core phenomena mentioned, slightly different instances of the same plenomena could cause a parse failure. For reference, a small set of novel sentences were also parsed with the grammars, to determine their coverage outside the closed test.

The unpunctuated version of the grammar was prepared by removing all the features relating to specifically punctuational phenomena, and also removing explicit mention of punctuation marks from the rules. This, of course, left behind certain rules that were functionally identical, and so duplicate rules were removed from the grammar. Similarly for rules which performed the same function at different levels in the grammar (e.g. attachment of prepositions to the end of a sentence with a comma was also catered for by rules allowing prepositions to be attached to noun and verb phrases without a comma).

\section{RESULTS}

Results of parsing with the punctuated grammar wero very good, yielding, on average, a surprisingly small number of parses. The number of parses ranged from 1 to 520 , with an average of 38 . This average is unrepresentatively high, however, since only 4 sentences had over 50 parses. These were, in general, those with high numbers of punctuation marks, all containing at least 5 , as in (20). Ignoring the four smallest and four largest results then, the average number of parses is reduced to just 15 . Example (21) is more representative of parsing. On examination, a great number of the ambiguities seem to be due to inaccuracies or over-generality in the lexical tags assigned to words in the corpus. 'The word more, for example, is triple ambiguous as determiner, adjective and noun, irrespective of where it occurs in a sentence.

(20) (The sunlit weeks between were full of maids: Sarah, with orange wig and horsy teeth, was so bad-tempered that she scircely spoke; Maud was my hateful nurse who smelled of soap, and forced me to eat chewy bits of fish, thrusting me back to babyhood with threats of nappies, dummies, and the feeding botile.) 520 punctuated parses

(21) (More news about the reverend Sun Myung Moon, founder of the Unification Church, who's currently in jail for tax evasion: lse was awarded an honorary degree last weck by the Roman Catholic University of la Plata in Buenos Aires, Argentina.) 18 punctuated parses

Besides the ambiguity of corpus lags, a problem arose with words that had been completely mistagged. If these caused the parse to fail completely, the tag was changed in the development phase of the grammax, but even so, the number of complete mistags was rather small in the sul)-corpus used: around 10 words in the 50 sentences used.

Initial attempts at parsing the corpus subset using the unpunctuated version of the grammar wero unsuccessful on even the most powerful machine available. This was duc to the failure of the machine to represent all the parses simultaneously when unpacking the parse forest produced by the chart parser. A special section of code written for the GDL (grateful thanks are due to Joln Carroll for supplying this piece of code) to estimate the number of individual parses represented by the packed parse-forest showed that for all but the most basically punctuated sentences, the number of parses was ridiculously huge. The figure for the sentence in (21) was in excess of $6.3 \times 10^{12}$ parses! Even though this estimate is an upper bound, since eflects of feature value percolation cluring unpacking are ignored, it has been fairly accurate with most grammars in the past and still inclicales that rather too many parses are being produced! Not all sentences produced such a massive number of parses: the sentence in (22) yielded only 1.92 parses witl the unpunctuated grammar which was by fior the smallest number of unpunctuated parses. Most sentences that managed to pass the estimalion process produced between $10^{6}$ and $10^{\circ}$ parses.

$$
\begin{gathered}
\text { (Protestants, lowever, are a tiny minority in } \\
\text { Argentina, and the delegation won't be } \\
\text { including a Roman Catholic.) } \\
\text { 9 punctuated parses }
\end{gathered}
$$

On examination of the grammar and the corpus, it is possible to understand why this has happened. 'The punctuated grammar had to allow for sentences including comma-delimited noun phrases adjacent to undelimited noun phrases, as illustrated by the rules (15) and (16). These are relatively easy to mark and recognise when the punctuation is available. However, 
without punctuational clues, and with the underspecific tagging system, any compound noun could appear as a set of delimited noun plirases with the unpunctuated grammar.

Therefore the unpunctuated grammar was further trimmed, to such an extent that parses no longer accurately reflected the linguistic structure of the sentences, since, for example, comma clelimited noun phrases and compound nouns became indistinguishable. Some manual preparation of the sentences was also carried out to prevent the reoccurrance of simple, but costly, misparses.

The results of the parse now became much more tractable. For basic sentences, as predicted, there was little difference in the performance of punctuated and unpunctuated grammars. Results were within an order of magnitude, showing that no significant advantage was gained through the use of punctuation. 'l'he sentences in (23) and (24) recoived 1 and 11 parses respectively with the umpunctuated grammar.

$$
\begin{gathered}
\text { (Well, just recently, a day conference on } \\
\text { miracles was convened by the research } \\
\text { scientists, Christian Fellowship.) } \\
4 \text { punctuated parses }
\end{gathered}
$$

(The assembly will also be discussing the UK immigration laws, Ilong Kong, teenagers in the church, and of course, church unity schenes.) 2 punctuated parses

(They want to know whether, for instance, in a scientific age, Christians can really believe in the story of the feeding of the five thousand as described, or was the miracle that those in the crowd with food shared it with those who had none?) 24 punctuated parses

For the most complex sentences, however, the number of parses with the mpunctinated grammar was typically more than two orders of magnitude higher than with the punctuated grammar. The sentence in (25) had 12,096 unpunctuated parses.

l'arsing a set of ten previously unseen punctuationally complex sentences with the punctuated grammar resulted in seven of the ten being unparsable. The other three parsed successfully, with the number of parses falling within the range of the results of the first part of the investigation. The parse failures, on examination, were due to novel punctuational constructions occurring in the sentences which the grammar had not been designed to handle. Parsing the unseen sentences with the unpunctuated grammar resulted in one parse liailure, with the results for the other 9 sentences reflecting the previous results for complex sentences.

\section{DISCUSSION}

This investigation seems to support the original premise - that inclusion and use of punctuational phenomena within natural language syntax can insist the general aims of natural language processing.
We have seen that for the simplest sentences, use of punctuation gives us little or no advantage over the more simple grammar, but, conversely, does no harm and can reflect the actual linguistic construction a litlle more accurately.

for the longer sentences of real language, however, a grammar which makes use of punctuation massively outperforms an otherwise similar grammat that, ignores it. Indeed, it is difficult to see how any grammar that takes no notice of punctuation could ever become successful at analysing such sentences unless some huge amount of semantic and pragmatic knowledge is used to disambiguate the analysis.

However, as was shown by the attempt at, parsing the novel sentences, knowledge of the role of punctuation is still severely linited. T'he grammar only performed reliably on those punctuational phenomena it had been designed with. Unexpected constructs cansed it to fail totally. Therefore, following the recognition that punctuation can play a crucial role in matural language syntax, what is needed is a thorough investigation into the theory of punctuation. Then theoretically based analyses of punctuation can play a full and important part in the analysis of language.

\section{ACKNOWLEDGEMENTS}

This work was carried out uncler Esprit Acquilex-II, 13RA 7315, and an LSRC Researel Studentship, R00429334171. 'Tlanks for instructive and helpful comments to 'Ted Briscoe, Joln Carroll, Robert Dale, Henry 'Thompson and anonymous CoLing reviewers.

\section{REFERENCES}

Briscoe, F J and N Waegner (1992). "Robust Stochastic Parsing Using the Inside-Outside Algorithm." In Proceedings, AAAI Workshop on Statistically-based NIP Techniques, Sar Jose, CA. Carroll, J; E J Briscoe; and C Grover (1991). " $\Lambda$ Development linvironment for Large Natural langnage Grammars." 'Technical Report 233, Cambridge University Computer Iaboratory.

Ditle, R (1991). "lixploring the Role of Punctuation in the Signalling of Discourse Structure." In Procedings, Workshop on Text Representation and Domain Modelling, 'T'. U. Berlin, pp110-120.

Dale, $R$ (1990). " $\Lambda$ Rule-based approach to Computer- $\Lambda$ ssisted Copy Ecliting." Computer Assisled language Learning, 2, pp59-67.

Itumphreys, R L (1993). "Book Review: 'T'he Linguistics of Punctuation." Machine Translation, 7.

Markwardt, A H (1942). Introduction to the English Language, Oxford University l'ress, New York.

Nunberg, G (1990). The Linguistics of Punctuation, CSIJ I,echure Notes 18, Stanford, CA.

'Taylor, I, J and G Knowles (1988). Manual of Information to Accompany the SEC Corpus, University of Lancaster. 